\title{
Electrorefraction beyond excitonic resonance in a GaAs/AlGaAs multiple quantum well structure in the Franz-Keldysh geometry
}

\author{
M. Wichtowski* \\ Faculty of Electrical Engineering, West Pomeranian University of Technology, al Piastów 17, 70-310 Szczecin
}

Received November 06, 2020; accepted December 18, 2020; published December 31, 2020

\begin{abstract}
The Kramers-Kronig relations were used to estimate electrorefractivity near the edge of the exciton absorption peak of a $\mathrm{GaAs} / \mathrm{AlGaAs}$ photorefractive quantum well (PMQW) structure working in the Franz-Keldysh geometry. It was shown that for both TE and TM polarizations the change of the refractive index under an applied electric field is at least an order of magnitude greater than in bulk semiinsulating GaAs due to the classical Franz-Keldysh effect.
\end{abstract}

The photorefractive (PR) effect relies on the change in the optical properties of material under the influence of light with a nonuniform intensity distribution [1]. Among several classes of photorefractive (PR) materials semiconductors such as semi-insulating $\mathrm{GaAs}$ are characterized by fast response to changes in illumination and high sensitivity in the near infrared range. Their disadvantage is, however, the low values of electrooptic (EO) coefficients $(\sim 1 \div 10 \mathrm{pm} / \mathrm{V})$. One way to enhance the EO response in bulk GaAs is to exploit optical resonance phenomena such as a field-induced electroabsorption (EA) caused by the Franz-Keldysh effect, which produces exponential tails in the absorption edge towards longer wavelengths [2, 3]. Electroabsorption is associated with the changes in the refractive index (electrorefraction ER) through the Kramers-Kronig relation [1, 3]. A Comprehensive study of the photorefractive effect in semi-insulating GaAs near the band edge has been carried out in [4], where the combination of the conventional linear EO effect and the quadratic Franz-Keldysh effect have been considered. In that case changes in the refractive index $\Delta n(\lambda, E)$ and in the extinction coefficient $\Delta \kappa(\lambda, E)=\alpha \lambda /(4 \pi)$ can be written as

$$
\begin{aligned}
\Delta n(\lambda, E) & =-(1 / 2) n_{0}^{2}\left[r_{1}(\lambda)|E|+s_{1}(\lambda) E^{2}\right], \\
\Delta \kappa(\lambda, E) & =-(1 / 2) n_{0}^{2}\left[r_{2}(\lambda)|E|+s_{2}(\lambda) E^{2}\right],
\end{aligned}
$$

where $n_{0}$ is the average refractive index, $r(\lambda)$ and $s(\lambda)$ denote, respectively, the effective linear and quadratic EO coefficients - see Fig.1.

\footnotetext{
*E-mail: marekw@zut.edu.pl
}
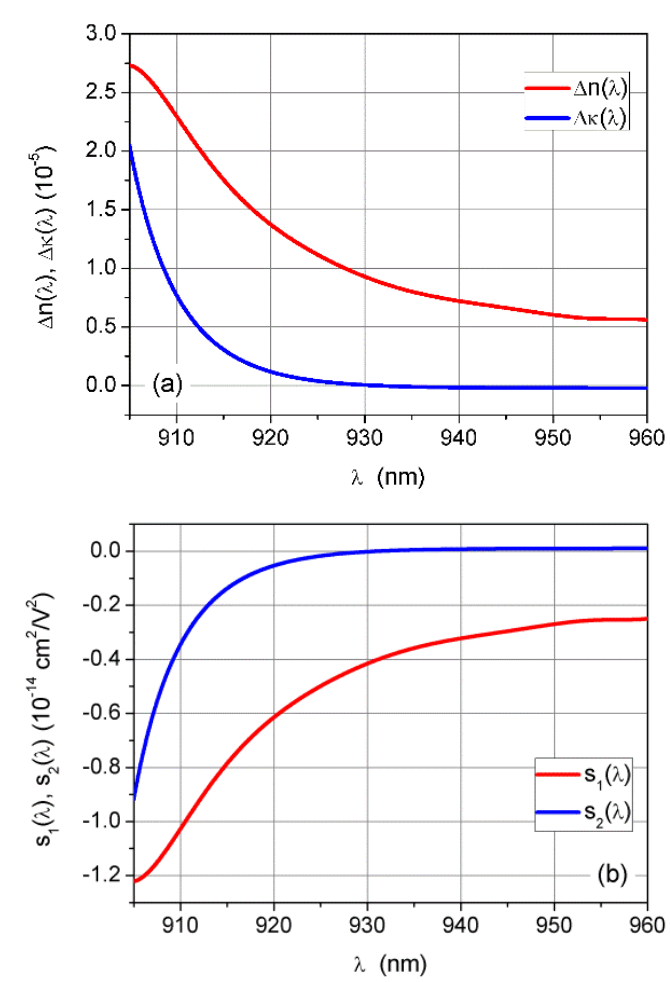

Fig. 1. (a) Change in the refractive index and the extinction coefficient due to the F-K effect for the field $E=10 \mathrm{kV} / \mathrm{cm} \mathrm{[4],} \mathrm{(b)} \mathrm{the} \mathrm{quadratic}$ EO coefficients obtained from the plot (a) and Eqs. (1). Errors in $\Delta n, \Delta \alpha$ and $s$ values have been evaluated up to $\sim 20 \%$

For photorefractive applications it is desirable that the value of $\Delta n$ should be as large as possible with a low value of $\alpha$. Assuming $\lambda=910 \mathrm{~nm}$, we find that for GaAs $\left(n_{0}=3.55, r=r_{41}=1.2 \times 10^{-10} \mathrm{~cm} / \mathrm{V}, s_{1} \approx 10^{-14} \mathrm{~cm}^{2} / \mathrm{V}^{2}\right)$ under the biasing field $E=10 \mathrm{kV} / \mathrm{cm}$ linear and quadratic changes in the refractive index are $\Delta n_{\text {lin }} \sim \Delta n_{\text {quadr }} \sim 10^{-5}$. For stronger fields the square effect begins to dominate over the linear one.

Significantly stronger nonlinear effects are observed in semiconductor photorefractive multiple quantum wells (PMQW) structures, where the electro-optic properties are enhanced by exciton resonances appearing as peaks in the absorption spectrum, which are sensitive to the electric field. This nonlinearity leads to strong EA and ER but is 
simultaneously related with the large absorption of light waves tuned to excitonic transitions. For this reason, PMQW structures are typically used as thin layers ( $1 \mu \mathrm{m})$ in transmission geometry. The electric field applied in the plane of QW's causes broadening and disappearing of excitonic peaks - the effect like that of the FranzKeldysh in conventional bulk semiconductors (for this reason such configuration is called the Franz-Keldysh geometry). For moderate electric fields $(<8 \mathrm{kV} / \mathrm{cm}) \Delta n$ and $\Delta \kappa$ exhibit approximately quadratic dependence on the electric field [1] which can be formally described by expressions (1) neglecting terms with $r$.

In the present work it is pointed out that despite numerous studies of exciton nonlinearity in MQW, according to the author's knowledge, the magnitude of ER below the exciton absorption edge for PMQW in the Franz-Keldysh geometry has not been investigated. The aim of this study is, using the transmission measurements of the PMQW GaAs / AlGaAs sample and the K-K relation, to estimate the strength of ER below the excitonic absorption threshold and to compare it with ER for bulk GaAs.
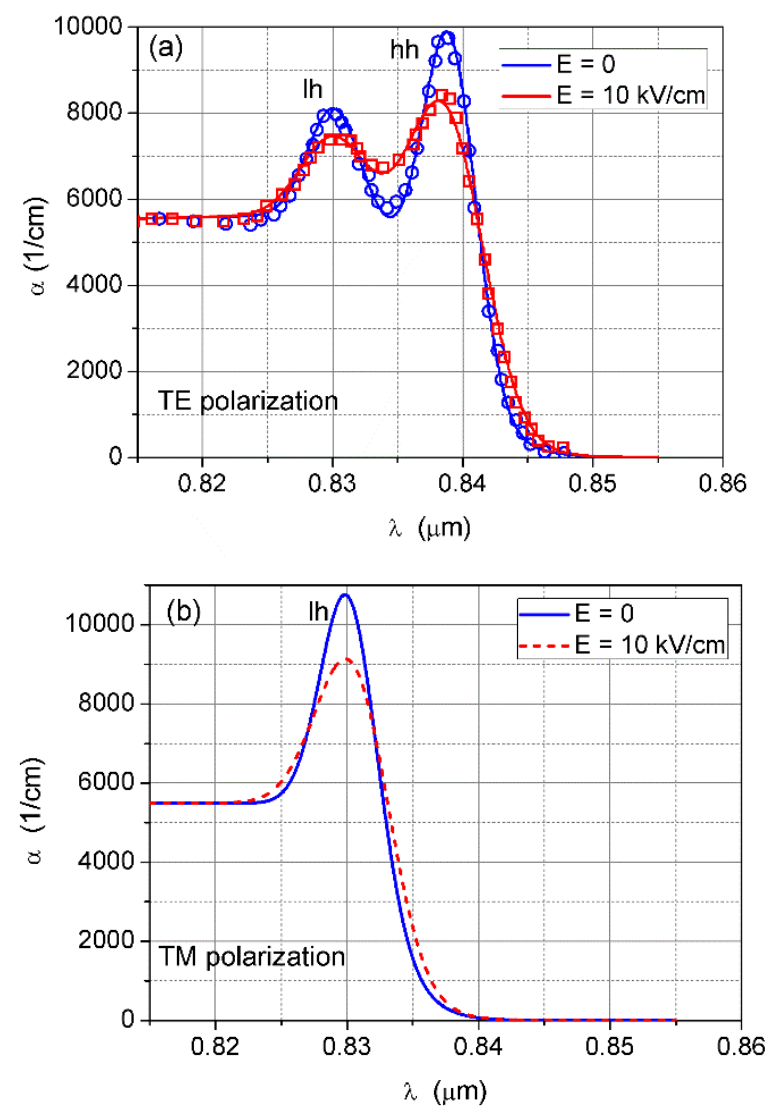

Fig. 2. Room-temperature absorption spectra of the $\mathrm{GaAs} / \mathrm{Al}_{0.3} \mathrm{Ga}_{0.7} \mathrm{As}$ PMQW sample for an applied field $E=0$ and $E=10 \mathrm{kV} / \mathrm{cm}(h h-$ heavy holes, $l h$ - light holes), (a) experimental spectra for the polarization TE, (b) theoretically estimated spectra for the polarization TM.
The investigated structure PMQW was composed of 60 periods of $7.5 \mathrm{~nm}-\mathrm{GaAs}$ wells and $10 \mathrm{~nm}-\mathrm{Al}_{0.3} \mathrm{Ga}_{0.7} \mathrm{As}$ barriers. The thickness of the AlGaAs barriers of $10 \mathrm{~nm}$ makes that quantum coupling between the wells weak and so the structure can be treated as a system of 60 isolated wells designed for exciton transitions to the $n=1$ states. The experimental absorption spectra at room temperature are shown in Fig. 2a. Above $\lambda=0.85 \mu \mathrm{m}$, both graphs become practically indistinguishable in terms of measurement accuracy. The electroabsorption was determined on the basis of the sample transmission spectra without an applied electric field and with a field of $10 \mathrm{kV} / \mathrm{cm}$. The absorption coefficient was calculated, taking into account the fact that the sample, together with the thin glass plate on which it was epoxied forms a Fabry-Perot cavity. The interference ripples occurring beyond the heavy hole exciton peak of the spectrum, which are not shown in Fig. 2a, reduce the accuracy of electroabsorption determination in this range. Nonetheless, it should be noted that according to the K-K formulas, if there is an unaccounted additional electroabsorption tail after the resonance peak, it will increase the ER in this spectral range. Therefore, it can be assumed that the results obtained in this way give an approximate lower limit on the value of $\Delta n$.

The change in the absorption coefficient due to applied electric field is given by $\Delta \alpha(\lambda, E)=\alpha(\lambda, E)-\alpha(\lambda, 0)$. In turn, the change in the refractive index $\Delta n(\lambda, E)=n(\lambda, E)-$ $n(\lambda, 0)$ may be calculated from $\Delta \alpha$ by using the KramersKronig relation $[1,3]$ :

$$
\Delta n(\lambda, E)=\frac{\lambda^{2}}{2 \pi^{2}} \mathrm{P} \int_{\lambda}^{\lambda_{2}} \frac{\Delta \alpha\left(\lambda^{\prime}, E\right)}{\lambda^{2}-\lambda^{\prime 2}} d \lambda^{\prime},
$$

where $\mathrm{P}$ is the main value of the integral and the integration runs in limits of non-zero EA.

It should be noticed that the absorption coefficient of MQW structures is dependent on the polarization of a light wave. Polarizations for which an electric field vector of the light wave is parallel or perpendicular to the QW layers will be called as TE and TM polarizations, respectively. For TM polarization, transitions for heavy holes are forbidden and only the enhanced exciton peak of light holes appears in the absorption spectrum [5, 6].

The spectrum of an absorption coefficient for the exciton transitions between the valence band and conduction band for electron-hole states $n=1$ can be expressed as [7]:

$$
\alpha(\mathrm{h} \omega)=C\left|p_{c v}\right|^{2} \Phi\left(E_{e x}-\mathrm{h} \omega\right),
$$

where $C$ is the constant dependent on the values of material parameters, the function $\Phi\left(E_{e x}-\hbar \omega\right)$ describes the profile of the exciton line, $E_{e x}$ is the exciton transition energy, $\left|p_{c v}\right|^{2}$ denotes the polarization dependent 
momentum matrix element. For TE polarization one gets $\left|p_{c v}\right|^{2}=(3 / 2) M_{b}^{2}$ and $\left|p_{c v}\right|^{2}=(1 / 2) M_{b}^{2}$ for the exciton of heavy holes $(h h)$ and light holes $(l h)$ respectively. For TM polarization one obtains $\left|p_{c v}\right|^{2}=0$ (for $h h$ ) and $\left|p_{c v}\right|^{2}=2 M_{b}^{2}$ (for $l h$ ), where $M_{b}^{2}$ is the matrix element for a bulk semiconductor $[3,8]$.

In a typical setup where the transmission geometry is used, measurements are carried out for TE polarization see Fig. 2a. Obtaining the absorption spectrum for TM polarization is more difficult and requires the geometry of a properly designed planar waveguide. Figure $2 \mathrm{~b}$ shows the theoretically determined TM spectrum taking into account the gain of the light hole resonance according to the above given values of $\left|p_{c v}\right|^{2}$. Dispersion curves for EA and ER in the wavelength range with low attenuation are presented in Fig. 3. As seen for TE waves, the requirement of low absorption is satisfied for $\lambda>850 \mathrm{~nm}$, whereas for TM waves - for $\lambda>843 \mathrm{~nm}$. However, it should be noted that the value of the residual absorption is unknown, hence conclusions can only be drawn as far as the ER is concerned. Referring to Fig. 3a we find that for both polarizations the changes in the refractive index lie in the range $(1 \div 5) \times 10^{-4}$. Note that it is about one order of magnitude more than the quadratic ER coefficient associated with the Franz-Keldysh effect in bulk semiinsulating GaAs. The estimated error in determining the $\Delta n$ and $\Delta \kappa$ may reach $20 \%$, but this does not change the main conclusion. It is worth indicating that the wavelengths of the excitation peaks and the absorption edges can be shifted spectrally by changing the well width. Moreover, the spectral shift can be made by changing the temperature of the sample with the temperature coefficient $\Delta \lambda / \Delta T \sim 0.4 \mathrm{~nm} / \mathrm{K}$ [9]. Note also that the radiation with wavelengths in this range is generated by semiconductor lasers based on heterostructures GaAs/AlGaAs or InGaAs/AlGaAs [7].

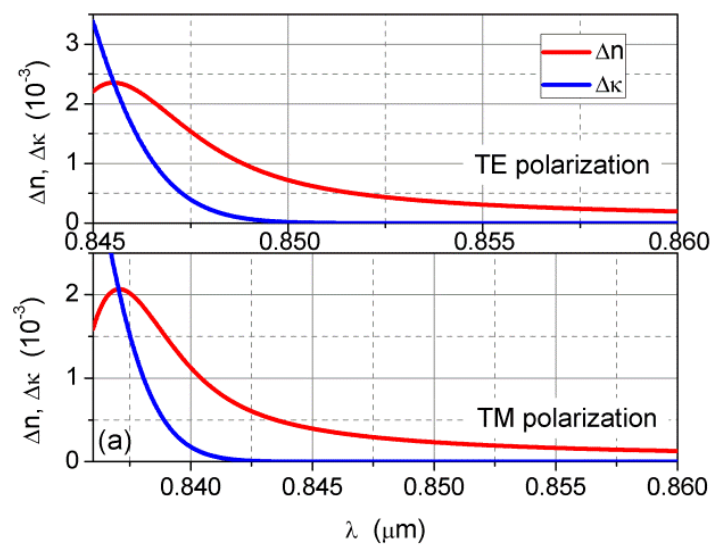

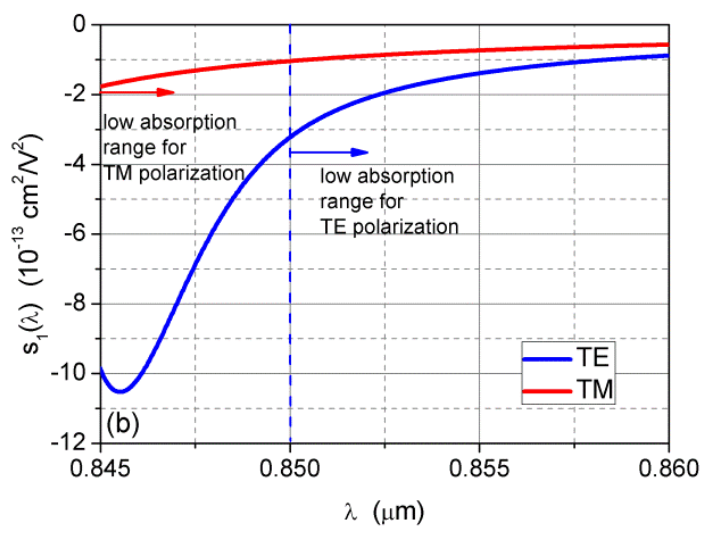

Fig. 3. Electroabsorption $(\Delta \kappa)$ and electrorefraction $(\Delta n)$ for an applied field $E=10 \mathrm{kV} / \mathrm{cm}$ beyond the exciton peaks in the range of low absorption (b) Spectra for quadratic EO coefficients $s_{1}(\lambda)$ for TE and TM polarizations obtained based on Fig. 3a and Eqs. (1).

Dispersion curves for quadratic EO coefficients $s_{1}(\lambda)$ and $s_{2}(\lambda)$ corresponding to ER and EA are presented in Fig. 3b. These curves should be compared with plots in Fig. 1b. Electrorefraction on the level of $10^{-4}$, although small compared to the average refractive index $n_{0} \approx 3.5$, may be sufficient in practical applications such as a grating-assisted waveguide coupler with PMQW, considered in [9]. Summarizing, it has been shown that the electrorefraction coming from the broadening and lowering of the exciton peaks (the "excitonic FranzKeldysh effect") is essentially much stronger in comparison to the near-band edge ER associated with the classical Franz-Keldysh effect for bulk semi-insulating GaAs. The wavelengths for the considered sample PMQW cover the approximate range of $\lambda>850 \mathrm{~nm}$ for TE polarization and $\lambda>843 \mathrm{~nm}$ for TM polarization, respectively.

\section{References}

[1] D.D. Nolte, M.R. Melloch in: Photorefractive effects and Materials, ed. by D.D. Nolte (Kluwer, Dordrecht 1995).

[2] T.E. Van Eck, L.M. Walpita, W.S.C. Chang, H.H. Wieder, Appl. Phys. Lett. 48, 451 (1986).

[3] P.K. Basu, Theory of Optical Processes in Semiconductors, ch.7 (Oxford University Press 2003).

[4] A Partovi. E.M. Garmire, J. Appl. Phys. 69, 6885 (1991).

[5] J.S. Weiner, D.A.B. Miller, D.S. Chemla et. al., Appl. Phys. Lett. 47, 1148 (1985).

[6] D.S. Chemla, D.A.B. Miller, J. Opt. Soc. Am. A 21155 (1985).

[7] S.L. Chuang, Physics of Photonic Devices, ch. 14 (2nd Ed. New Jersey, Wiley \& Sons 2009)

[8] E. Miśkiewicz, A. Ziółkowski, M. Wichtowski, E. Weinert - Rączka, Opt. Mat. 89, 231 (2019).

[9] E. Weinert-Rączka, R. Iwanow, Acta Phys. Pol. A 95, 813 (1999). 